\title{
VIH-Sida : éthique du soin, de la recherche et accès aux traitements
}

$>$ Le VIH-sida doit se comprendre comme un défi dont on ne peut atténuer ni la violence, ni la gravité. On peut toutefois constater que cette pandémie a suscité et édifié de nouvelles formes de

\section{Emmanuel Hirsch} solidarités qui ont bouleversé nos repères en termes de soins et de santé publique. Ces quelques acquis des années sida éclairent autrement la figure de la personne malade et nous permettent de comprendre avec d'autres références la dimension politique de la santé publique. Les notions de respect, de solidarité et d'équité se comprennent dans le cadre d'un partenariat, et parfois même d'une alliance, thérapeutique. La relation de soin s'est située dans une perspective jusqu'alors inédite. Nos avancées révèlent davantage les injustices dans l'accès aux traitements et fixent l'horizon de nos responsabilités morales à l'égard de toute personne malade. La lutte contre le sida doit désormais se comprendre en termes de justice et de moyens investis dans le partage des acquis, tout particulièrement auprès des communautés les plus vulnérables. Les situations d'extrême précarité, les questions relatives aux conditions d'accès aux soins, à l'information, aux protocoles expérimentaux comme aux traitements et la qualité d'existence des personnes constituent autant d'aspects tangibles des défis qu'il nous faut considérer, aujourd'hui encore, en termes d'urgence vitale. <

\section{Une certaine exemplarité}

Les personnes affectées par le VIH-sida ont su découvrir au sein du milieu associatif et auprès de la communauté soignante la qualité et la force d'une mobilisation très exceptionnelle. On doit pour beaucoup à cette solidarité pratique l'émergence d'une figure différente de la personne malade, impliquée dans le soin, la recherche, le soutien des autres et l'expression publique de droits et

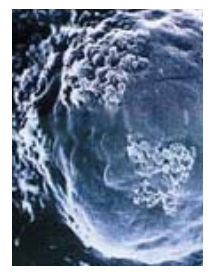

de devoirs qui ont conféré une certaine exemplarité aux années sida.

Dans l'approche éthique des réalités humaines liées au VIH-sida, le respect de l'autonomie et de la responsabilité de la personne accompagne fort justement la mise en œuvre des dispositifs de prévention, de dépistage et d'accès aux soins en référence aux principes de la Déclaration universelle des droits de l'homme. En 1988, une circulaire [1] note que «le gouvernement français a exclu jusqu'à présent toute politique de dépistage systématique ou obligatoire (à l'exception du dépistage obligatoire sur tous les dons d'organes et de sang), pour lui préférer une politique de responsabilisation fondée sur l'information d'une part, le dépistage volontaire d'autre part ». Selon un communiqué de l'Organisation Mondiale de la Santé [2], celle-ci est «préoccupée par la discrimination à l'encontre des personnes infectées par le VIH et des malades du sida, qui entrave les efforts faits pour freiner la propagation de cette maladie. Tous les jours, dans le monde entier, des personnes infectées et des malades du sida sont victimes de mesures discriminatoires [...]. En mai 1992, l'Assemblée mondiale de la Santé a souligné le lien existant entre les considérations de santé publique et la protection des droits des personnes infectées par le VIH et des malades du sida. L'Assemblée a adopté une résolution reconnaissant qu'aucune considération de santé publique ne saurait légitimer des mesures de lutte contre le sida attentatoires aux droits des individus, et notamment des mesures instaurant un dépistage obligatoire. L'Assemblée a demandé aux États membres de 
l'OMS de s'opposer à la discrimination dont sont victimes les personnes et les groupes particuliers que l'on sait infectés par le VIH ou que l'on soupçonne de l'être. Elle s'est engagée à ce que les États membres adoptent une attitude humanitaire face au VIH/sida pour que la santé publique n'ait pas à pâtir de la discrimination et de la stigmatisation [...] ». Un consensus s'est établi autour de valeurs fortes, quelles que soient les contraintes et les menaces qui n'ont pas manqué de peser depuis les premiers temps de l'épidémie. Les notions de respect, de solidarité et d'équité se comprennent dans le cadre d'un partenariat, et parfois même d'une alliance thérapeutique. La relation de soin s'est située dans une perspective jusqu'alors inédite.

Confronté aux modes de transmission du virus et à ses conséquences trop souvent discriminatoires, il convenait de développer une pédagogie de la responsabilité partagée. L'approche éthique s'est ainsi imposée dans l'espace politique en termes de résistance et de vigilance, suscitant les formes les plus diverses d'un combat qui s'est progressivement imposé dans le champ de la santé publique: «Révélatrice de la qualité des valeurs que nous prétendions tant défendre - tolérance, respect de la vie privée, solidarité, l'épidémie du sida place les sociétés démocratiques face à des choix décisifs. La façon de gérer cette épidémie ne manquera pas de marquer profondément la société que nous laisserons à nos enfants. En choisissant les impératifs de l'État de droit pour résoudre la question du sida, c'est notre propre liberté que nous choisissons » (Daniel Borillo [3]).

Au début, il s'agissait avant tout de préserver ou de reconstituer un lien social, de dénoncer les attitudes de rejet, d'inciter la communauté soignante à une plus juste attention. Une infirmière en témoigne: « C'est tout le service qui était agressé par ce mal qui résistait à tout. On notait les progrès les plus infimes, on comptait les indices d'amélioration les plus imperceptibles. À ce stade d'attention à l'autre, on devine tous ses besoins, on peut précéder ses envies, apaiser ses exigences » [4]. De leur côté, il appartenait aux pouvoirs publics d'exercer autrement, selon des principes affirmés, les responsabilités qu'il leur revenait d'assumer. Claude Évin, ministre de la Santé, précise ses positions. «Cette lutte, j'entends la mener au nom et pour certaines valeurs: la solidarité, le refus de l'exclusion, la responsabilisation. Ceci n'est pas de l'angélisme. L'affirmation de ces valeurs a permis une mobilisation civique et sociale qui n'aurait pas été atteinte autrement. Ce sont ces valeurs qui donnent aux malades l'espoir nécessaire pour se battre et à nos concitoyens la volonté d'agir contre la maladie » [5].

En France, on peut émettre une appréciation plutôt favorable à l'égard des multiples évolutions bien souvent imposées par des pressions argumentées, sans pour autant se satisfaire d'acquis toujours fragiles et partiels. Dans les faits, l'accueil au sein de l'hôpital, la relation entre personnes malades et équipes médicosoignantes, l'accès à l'information, la participation aux décisions en matière de recherche et de traitement, le suivi notamment au sein de réseaux ville-hôpital ainsi que l'accueil des personnes en situation de vulnérabilité sociale et de marginalité représentent autant d'avancées d'une portée très significative. La réponse au phénomène épidémique s'est imposée au sein des systèmes de soins qui ont su concevoir et soutenir des réponses d'autant plus appropriées qu'elles procédaient d'un sentiment d'urgence et de l'exigence profonde de soutenir une indispensable critique des pratiques en vigueur. Claude Got relève ainsi dans son rapport sur le sida de 1989: «Nous devons nous demander si nous avons une conception de notre système de soins à la hauteur de l'enjeu humain que représente l'assistance morale et physique de l'individu malade »[6]. En ce qui la concerne, la société civile n'a témoigné d'une conscience de ses responsabilités que dans la brève période où elle éprouvait la proximité d'une menace, atténuée en 1996 par l'avènement des trithérapies qui intègrent le traitement du VIH-sida dans une sphère dès lors indifférenciée à prévalence biomédicale.

Les associations de lutte contre le sida portent la mémoire douloureuse de ces personnes anonymes qui se sont efforcées de conférer à leur lutte contre la maladie la dimension d'un acte politique. Vivre avec la maladie constitue l'affirmation d'un engagement et d'une revendication, mais également d'une confiance témoignée à une société susceptible d'être préoccupée des siens. C'est ainsi, dans cette utopie militante, que la personne malade s'est imposée dans toute sa dignité.

Devenu acteur, voire activiste, le malade affecté par le VIH-sida impose des principes qui rejoignent dans bien des cas les positions défendues par les soignants et les chercheurs. Tous sont associés dans ce qu'ils considèrent comme un défi et une lutte qui leurs sont communs. La Déclaration universelle des droits des malades du sida et des séropositifs (voir Encadré) constitue une charte d'une valeur et d'une portée qui caractérisent une approche jusqu'alors jamais élaborée des droits socio-politiques reconnus à toute personne affectée par une maladie.

En 1990, les États Généraux Vivre le sida ${ }^{*}$ [7] instaurent un nouveau mode d'arraisonnement de la parole

\footnotetext{
* Par la suite, l'Association AIDES recueillera des témoignages sur Les 1001 façons
} de vivre avec son traitement [8] 
publique par les personnes malades, et le partage d'expériences légitimées au sein d'une communauté humaine rassemblée dans la maladie. «Nous sommes tous concernés par l'épidémie du sida, en traitement, séropositifs ou proches de personnes atteintes, et nous avons des choses à dire [...]. Montrer que l'on est des gens, et que l'on ne laissera pas décider à notre place. Cette démarche nous réintègre dans l'histoire de l'épidémie, elle nous transforme, nous, patients, en acteurs - allant jusqu'à oser affirmer qu'ensemble, nous avons le pouvoir de dire ce qu'il nous faut pour mieux vivre, le pouvoir de décider de la prise en charge de notre maladie - y compris dans le choix des traitements médicaux qui nous sont proposés et l'élaboration des protocoles $^{\star \star} \gg$. Huit ans plus tard, la Ligne nationale contre le cancer organisera à son tour les premiers États Généraux des malades du cancer [9], reprenant, bien tardivement, des enjeux qui s'imposent et contestent des mentalités et des pratiques estimées dès lors inacceptables. Un déplacement ou une redistribution des légitimités et des compétences s'opère face à ce «fait social total », terme que l'ethnologue Marc Augé appliquera au sida [10], vécu dans l'immédiat, et qui excède le champ jusqu'alors imparti au soin. Le sida est pensé comme révélateur de dysfonctionnements et principe de transformation à comprendre dans une perspective résolument culturelle. Daniel Defert soutiendra: «Nos chercheurs et nos cliniciens ne la [l'épidémie] vaincront pas seuls; cela engage toute notre culture, notre attitude face à la discrimination, à la déchéance physique, à la sexualité, à la toxicomanie et à la mort. II faut des instruments intellectuels et un langage » [1]]. Le sida suscite de la part de ses différents protagonistes, investis d'une fonction souvent située aux marges de nos systèmes, l'invention d'une conception politique différente des missions du soin [12]. «Au premier rang de ces principes figure le souci de préserver la santé publique tout en respectant la dignité du malade et, plus largement, de la personne humaine, quelle que soit la nature ou l'origine de sa maladie. II s'agit là d'un principe imprescriptible, et c'est l'honneur et le devoir des sociétés civilisées que d'en garantir le respect en toutes circonstances $\gg[13]$.

Cette crise de l'autorité, de la distribution des légitimités, du savoir et des pouvoirs explique nombre de mutations intervenues dans le champ des pratiques soignantes. À bien des égards, la loi n 90-602 du 12 juillet 1990 relative à la protection des personnes contre les discriminations en raison de leur état de santé ou de leur handicap, ainsi que la loi $n^{\circ} 2002-303$ du 4 mars 2002

* * Notes de l'auteur relative aux droits des malades et à la qualité du système de santé, consacrent des acquis que l'on ne peut comprendre et intégrer qu'en les situant dans la dynamique de la politisation ou de la démocratisation de la gestion d'une maladie considérée comme une affaire publique.

Cela étant, il semble évident que nous ne pouvons pas nous limiter au seul constat de ces quelques avancées. Les droits de toute personne malade doivent être envisagés comme principes inaliénables et références indispensables à l'exercice de nos responsabilités. Dès lors, les situations d'extrême précarité, les questions relatives aux conditions d'accès aux soins, à l'information, aux protocoles expérimentaux comme aux traitements et la qualité d'existence des personnes constituent autant d'aspects tangibles des défis qu'il nous faut considérer, aujourd'hui encore, en termes d'urgence vitale.

\section{L’horizon de nos responsabilités}

En France, la communauté scientifique, médicale et soignante a su assumer, dans sa grande majorité, une implication évidente dans la recherche, les traitements et les soins. À juste titre, il est possible de considérer qu'auprès des malades et des associations, les professionnels ont dégagé les nouvelles perspectives d'une conception de la relation médicale. Elle associe, autant que faire se peut, la personne dans les décisions qui la concernent, témoignant d'un extrême souci de sa qualité de vie pour une prise en compte globale indispensable au suivi d'une pathologie à ce point lourde et complexe. Selon Louis René [14], alors président du Conseil national de l'ordre des médecins, « La diffusion mondiale du VIH est un défi pour la communauté médicale; mais les règles professionnelles gardent toute leur valeur, aujourd'hui comme dans les siècles passés. Sous-estimer la situation actuelle - et à venir - serait une faute, de même que prôner de fausses solutions comme de donner pour efficaces des thérapeutiques charlatanesques, de préconiser des mesures de prévention s'il est établi qu'elles sont illusoires ou chimériques, d'exclure de la société tel groupe de population. Les médecins, et tous ceux qui à un titre quelconque participent aux soins, doivent avoir le souci permanent, lancinant, d'informer en termes adaptés sur la maladie, sur les mesures prophylactiques, nécessaires et utiles. Mais comment le médecin pourrait-il espérer s'acquitter de ces diverses missions si par son attitude personnelle, et ses propos, il ne témoigne pas en permanence de son respect de l'intimité, de la dignité, de la personnalité de celui qui s'est confié à lui pour être soigné, aidé, parfois accompagné jusqu'au terme de sa vie? ». 
L'accès aux traitements les mieux adaptés constitue actuellement un acquis difficilement contestable; à cet égard, les pouvoirs publics et l'institution hospitalière ont témoigné d'un sens évident de la justice sociale dans la mesure où les arguments économiques n'ont que rarement constitué un obstacle. Des lignes budgétaires spécifiques ont été attribuées au traitement du sida, ce qui a notamment favorisé la mise à disposition de molécules innovantes, la quantification de la charge virale, mais également la création de structures et de fonctions visant à l'amélioration des conditions d'accueil et de suivi à l'hôpital et en ville. La spécificité de telles approches a contribué à une meilleure prise en considération de la nature toujours singulière de l'expérience humaine de la maladie grave. À titre d'exemple, les conditions de réalisation d'une sérologie, d'annonce d'un résultat, de concertation et de consentement dans le choix d'un traitement, ainsi que d'inclusion dans un protocole de recherche ont gagné en rigueur et en justesse dès lors que la personne est considérée comme partenaire dans son soin.

Encore est-il nécessaire, s'agissant du sida comme d'autres maladies chroniques, que la personne puisse bénéficier du soutien social qu'impose souvent une précarisation de sa situation matérielle. Aujourd'hui, cette pathologie affecte majoritairement des personnes désocialisées qu'il conviendrait d'accompagner plus concrètement dans le combat qu'elles mènent contre la maladie. Si le bénéfice espéré des trithérapies permet à certains malades d'envisager différemment leur devenir, ainsi qu'une possible réinsertion sociale, il semble évident que le retour à l'emploi s'avère pour le moins peu évident dans le contexte actuel. Notre responsabilité à l'égard de la personne malade ne peut en aucun cas se limiter à la seule mise à disposition des traitements. D'autres mesures s'imposent pour qu'elle puisse vivre son existence au-delà de l'espace restrictif de sa maladie. Il est, sur notre territoire national, des personnes dépourvues de tout droit. Elles éprouvent à un niveau de violence insoutenable le scandale de la maladie. Qu'en est-il des droits de la personne humaine et de nos obligations à son égard dès lors qu'on lui conteste ainsi les conditions mêmes de sa survie? Comment admettre une telle relégation, une telle exclusion du soin, là où la déontologie médicale relève en toutes circonstances de principes universels estimés intangibles?

De même, comment ne pas considérer comme un échec inacceptable notre incapacité à partager le bénéfice des traitements avec les personnes affectées par le VIH-sida dans des régions du monde dont le système de santé carentiel et l'insolvabilité en font de véritables parias? Le VIH-sida a certainement contribué au profond mouve- ment de mobilisation observé depuis les années quatrevingt dans le champ du soin. Le caractère très relatif des options thérapeutiques, jusqu'à la mise sur le marché de la zidovudine en 1987, a favorisé la redistribution de positions, légitimités et pouvoirs jusqu'alors incontestés. II serait discutable d'y voir, de manière indifférenciée, une plus haute conscience d'enjeux éthiques dans la relation de soins là où les décisions ont bien souvent été adoptées en se référant au principe du «moindre mal ». L'accueil des personnes socialement vulnérables au sein des hôpitaux, la reconnaissance des droits de la personne marginalisée par l'usage de drogues, l'incarcération ou la prostitution, tout comme nos attitudes face à la douleur ou en situation de fin de vie, relèvent d'un devoir de non-abandon. C'est plutôt cette exigence de souci témoigné à l'autre et de solidarité active qui a contribué à l'émergence d'une nouvelle culture du soin instaurée - ne convient-il pas de le rappeler? - seulement par quelques visionnaires alors en marge des institutions.

On ne peut encore affirmer que le sida constitue un paradigme compris dans la globalité de ses significations, dès lors que ses acquis ne sont pas considérés comme une référence défendue en toute circonstance et particulièrement au plan international. Nos avancées révèlent davantage les injustices dans l'accès aux traitements et fixent l'horizon de nos responsabilités morales à l'égard de toute personne malade. En termes de défi, l'éthique du soin impose de transposer nos savoirs, nos compétences et nos moyens dans les pays progressivement anéantis par l'extension de la pandémie. Jonathan M. Mann, directeur du programme mondial de lutte contre le sida, le rappelait lors de l'inauguration de la Ve Conférence internationale sur le sida de 1989 [15]: « Nous devons être assez sages pour refuser de séparer la condition de quelques-uns du sort du plus grand nombre. Nous devons combattre la négligence et l'indifférence pour le sort des peuples et des nations. [...] Concernés par le sida, nous sommes entraînés à la solidarité. Cette solidarité nous donne la confiance nécessaire pour continuer de regarder le sida en face et être assurés que nous ne nous détournerons pas ».

Le 7 juillet 2002, UNAIDS, I'UNICEF et USAID rendaient public leur rapport consacré aux orphelins du sida [16] : 13,4 millions d'enfants de moins de 15 ans ont perdu leurs parents des suites de la maladie; d'ici 2010 , ils seront 25 millions!

\section{Un combat pour la vie}

Le contexte actuel peut être perçu selon différentes lectures et en fonction de notre situation sociale et géographique dans le monde. La confrontation de réalités 
contradictoires du VIH-sida en devient d'autant plus intolérable qu'elle dénonce notre faculté d'atténuer la force d'une indignation plus justifiée que jamais. La lutte contre le sida doit désormais se comprendre en termes de justice et de moyens investis dans le partage des acquis, tout particulièrement auprès des communautés les plus vulnérables. II semble évident qu'une telle exigence ne peut se satisfaire des pétitions de principe ou d'intentions, aussi généreuses soient-elles, indifférentes aux facteurs socio-politiques qui accentuent cette tragédie. La corruption des systèmes, l'absence de vie démocratique, les déplacements des populations, la relégation sociale de la femme et des enfants, les pauvretés et détresses humaines constituent autant de défis et d'obstacles dans la mise en $œ u v r e$ des politiques de santé publique. Alors que force est de constater chaque année l'insoutenable progression du nombre des personnes affectées par le VIH-sida, certains pays ont su inverser la courbe épidémique et inventer une approche qui constitue une référence. Dans son édition du 28 janvier 2001, The New York Times Magazine rend hommage à la mobilisation d'une nation dans le contexte des pays en développement: «Le Brésil a démontré que, armé de la volonté de lutter, un gouvernement peut faire davantage que de s'asseoir pour observer le désert progresser. » Si 115000 Brésiliens bénéficient aujourd'hui de l'accès à une gamme de quinze antirétroviraux, c'est parce que ce pays a considéré ses obligations face au VIH-sida en termes d'engagement politique et de respect inconditionnel des droits de la personne. En 2001, le Brésil [17] dénombrait 610000 personnes vivant avec le virus (dont 220000 femmes et 13000 enfants), plus de 220000 cas déclarés de sida, 8400 décès et 37000 orphelins. Dès 1994, il s'est impliqué à la fois dans le soutien des personnes affectées par le VIH-sida et dans la promotion d'une information à grande échelle. Cent cinquante millions de dollars américains (US \$) ont été investis dans cet effort (60 financés par la Banque mondiale et 90 par l'État) dans le cadre d'un partenariat entre les instances publiques et la société civile. La loi de 1996 sur l'accès aux traitements ainsi que la nouvelle Constitution de 1998 consacraient la reconnaissance d'un droit universel à la santé relevant des devoirs et missions de l'État. Si les données quantifiées permettent de constater l'impact du deuxième programme sida (1998-2001), il convient toutefois de chercher à comprendre la signification et la portée d'une démarche qui semble concilier efficacité et rigueur morale dans un contexte social pourtant défavorisé. L'accès universel et gratuit aux traitements a réduit de $50 \%$ la mortalité et de $80 \%$ les hospitalisations, produisant sur les cinq dernières années une éco- nomie d'un milliard de dollars US. Les dispositifs sanitaires proposés aux malades répondent à la diversité des situations, privilégiant des suivis compatibles avec le maintien d'une vie sociale. Du point de vue de la prévention, en 2001, 1,6 millions de personnes ont bénéficié d'un dépistage volontaire, anonyme et gratuit. Chaque année, plus de 600 millions de préservatifs sont mis à la disposition des populations.

Dans une publicité se félicitant de la reconnaissance exprimée par les Nations-Unies au programme de prévention du sida, considéré comme le meilleur des pays en développement, le gouvernement brésilien ne manque pas d'aborder franchement un des éléments déterminants de ses acquis: «La fabrication au plan local de nombreux médicaments utilisés dans les traitements contre le sida ne constitue pas une déclaration de guerre contre les firmes pharmaceutiques. Il s'agit simplement d'un combat pour la vie ».

II est certain que les accords passés par les autorités brésiliennes avec les industriels leur permettent d'accéder de manière favorable à des médicaments génériques. Cette approche volontariste confère une indiscutable pertinence à la résolution adoptée par les Nations-Unies en juillet 2001 [18]. La politique tarifaire des firmes pharmaceutiques dans les pays en développement est de nature à favoriser l'accès aux antirétroviraux, avec pour impact immédiat une diminution de la mortalité et la capacité de développer des politiques de santé publique susceptibles de limiter la progression de la pandémie et d'en atténuer les effets socio-économiques. En novembre 2001, les 142 états membres de l'Organisation mondiale du commerce (OMC) réunis à Doha ont signé une déclaration permettant à certains pays de produire ou d'importer des génériques*. C'est dire que s'impose, certes avec encore trop de réticences et de lenteur, une conception différente de nos responsabilités internationales dans le champ de la santé. La perception de l'urgence, face à des situations catastrophiques qui semblent échapper à toute possibilité de régulation, semble désormais unir des partenaires, jusqu'alors opposés, dans une conscience plus résolue de leurs devoirs et de leurs missions. À l'épreuve d'une telle réalité, la pression des activistes et l'intelligence des réalisations communautaires sur le terrain ébranlent les logiques et imposent un nouveau mode de pensée. Certains pays comme la Russie ne semblent pourtant pas l'avoir compris: dans ce pays, plus de 200000 personnes ont été contaminées par le VIH depuis trois ans. L'estimation la plus basse fait état de plus d'un million de personnes vivant avec le VIH-sida, 90 \% d'entre elles utilisant des drogues injectables. Trente-trois mille (33000) prisonniers sont séropositifs et n'ont que très 
rarement accès à un suivi médical. La politique discriminatoire à l'égard des personnes toxicomanes contribue à rendre inévitable la progression de l'épidémie.

En mai 2002, les autorités brésiliennes ont lancé un programme de coopération internationale visant à soutenir la lutte contre le VIH-sida dans trente pays en développement, y compris en transférant leur expérience en matière de fabrication de génériques: « Cette maladie révolutionnaire qui bouleverse les communautés à travers le monde en appelle à des mutations radicales aux plans individuel, communautaire, national et international » [19]. Il convient d'accompagner ces mutations radicales par une plus juste compréhension de leurs valeur et signification politiques au plan international.

\section{Urgence pratique}

Le $1^{\text {er }}$ décembre 1992, Boutros Boutros-Ghali, secrétaire général de l'ONU, intervient à l'occasion de la sixième Journée mondiale du sida: «Donnons à nos efforts une véritable dimension de guerre. Cela demande deux moyens: d'abord un investissement financier qui soit porté à la hauteur de la menace, et cela dans tous les pays du monde, en commençant par les plus riches. En second lieu, il faut un effort de coordination étendu à l'ensemble des savoirs médicaux ». Les notions de coordination, de cohésion et de cohérence, voire de solidarité, touchent également à l'un des aspects majeurs des stratégies menées contre le VIH-sida: celui de la recherche.

Dans ce domaine également, l'extrême proximité entre les membres d'associations militantes, les médecins et les chercheurs a produit de nouvelles formes de relations et de pratiques marquées par l'exigence de pragmatisme, de transparence et de concertation. En France, le groupe inter-associatif TRT5 intervient comme expert à tous les stades d'élaboration des protocoles. La loi n $88-1138 \mathrm{du}$ 20 décembre 1988 relative à la protection des personnes qui se prêtent à des recherches biomédicales, modifiée par les lois $n^{\circ}$ 90-86 du 23 janvier 1990, $n^{\circ} 91-73$ du 18 janvier 1991 et $n^{\circ} 94-630$ du 25 juillet 1994, détermine des règles; un contrôle a priori est exercé par les CCPPRB (Comités consultatifs de protection des personnes dans la recherche biomédicale).

Un tel dispositif réglementaire n'est que très rarement en vigueur dans les pays en développement, ce qui a incité les instances internationales à proposer des recommandations de nature à sauvegarder le droit des personnes dans l'accès à la recherche. À cet égard, la

* La Declaration on the TRIPS Agreement and Public Health concerne, outre le VIH sida, la tuberculose, la malaria et d'autres agents responsables d'épidémies. Le prix actuellement le moins élevé d'une trithérapie générique est de l'ordre de 200 \$ US, contre 150000 dans les pays développés.
Déclaration d'Helsinki témoigne dans sa dernière version d'un attachement particulier aux conditions d'exercice de la recherche expérimentale dans les contextes de vulnérabilité sociale [20]: «La recherche médicale est soumise à des normes éthiques qui visent à garantir le respect de tous les êtres humains et la protection de leur santé et de leurs droits. Certaines catégories de sujets sont plus vulnérables que d'autres et appellent une protection adaptée. Les besoins spécifiques des sujets défavorisés au plan économique comme au plan médical doivent être identifiés. Une attention particulière doit être portée aux personnes qui ne sont pas en mesure de donner ou de refuser elles-mêmes leur consentement, à celles qui sont susceptibles de donner leur consentement sous la contrainte, à celles qui ne bénéficieront pas personnellement de la recherche et à celles pour lesquelles la recherche est conduite au cours d'un traitement ».

Déterminer les critères indispensables au respect des principes universellement admis qui préservent les droits et les intérêts directs des personnes, ainsi que ceux de leur communauté, s'impose [21]. La demande de règles précises et transparentes, élaborées dans le cadre d'un partenariat, constitue également un impératif que rien ne saurait contester. Les notions de bienveillance et de justice développées dans le rapport Belmont [22] doivent être reconnues dans toute leur pertinence.

En ce qui concerne le VIH-sida, l'accès à un protocole de recherche équivaut dans certains pays à la seule possibilité de bénéficier d'un traitement. II importe de ne pas assimiler l'exigence justifiée d'un accès universel aux traitements présentant une efficacité avérée au bénéfice incertain d'une démarche à visée expérimentale. À titre d'exemple, les controverses relatives au placebo illustrent cet enjeu [23]. Du point de vue des bonnes pratiques, les indications doivent être appréciées de manière rigoureuse, dans la clarté, au cas par cas, en tenant compte des effets possibles sur la qualité de vie de la personne et de ses proches, mais aussi des préjudices qu'elle pourrait subir. Nous voilà renvoyés aux quelques considérations qui fondent et éclairent les principes éthiques de la conduite d'essais cliniques dans les pays en développement: respect des spécificités socio-politiques et culturelles du pays dans l'élaboration des stratégies; prise en compte des caractéristiques de chaque système de santé et définition concertée d'objectifs qui concilient l'intérêt direct des personnes et les enjeux de la santé publique. L'évaluation des modalités de réalisation de tout protocole par un comité d'éthique autonome, pluridisciplinaire et accessible s'impose. Elle concerne particulièrement les conditions d'expression et de reconnaissance effectives du respect de la personne: autonomie dans sa décision, information argumentée et communication continue, 
consentement explicite, confidentialité préservée au sein des structures hospitalières comme dans la sphère privée, engagements dans le suivi médico-social pendant et après l'essai. Le comité d'éthique (voir aussi [24]) doit être reconnu dans sa faculté d'apprécier les critères qui légitiment une recherche, la conception et la réalisation de la recherche du point de vue des bonnes pratiques, les avantages envisagés et les inconvénients possibles, le respect des principes d'équité et de justice distributive dans la définition des critères d'inclusion, les modalités d'information de la personne et de sollicitation de son consentement, prenant si nécessaire en considération la situation des personnes analphabètes, les possibilités énoncées de se retirer d'un essai sans conséquences préjudiciables et, enfin, les moyens mis en œuvre pour anticiper et accompagner les conséquences humaines et sociales des essais.

L'accès à la sérologie du VIH constitue la condition initiale d'une inclusion. II convient de respecter les conditions d'une pratique éthiquement acceptable: information adaptée, principe du volontariat, accompagnement de la démarche, confidentialité dans la remise du résultat et proposition d'un suivi global à l'annonce d'une sérologie positive. On constate que la situation de la personne au sein d'un réseau familial et communautaire relevant souvent du droit coutumier peut constituer une entrave à l'accès au test comme aux traitements. II semble donc essentiel de promouvoir une approche fondée sur la règle de droit qui se réfère à la Déclaration universelle des droits de l'homme et aux principes déontologiques des professionnels de la santé. Dans ce domaine, la Charte d'éthique de la recherche dans les pays en développement, présentée en mai 2002 par l'Agence nationale de recherches sur le sida (ANRS) [25] (consulter également [26]), constitue désormais un document de référence: «Les bonnes pratiques dans la conduite des recherches sont garantes de leurs valeurs éthiques. L'accès des personnes aux bénéfices attendus d'une recherche constitue un droit fondamental. La recherche doit servir les intérêts de la personne au sein de sa communauté ».

On le constate, sans en minimiser pour autant l'intérêt, les nombreux débats relatifs aux «meilleurs standards de soin » ne résistent que difficilement à l'analyse concrète des situations. Serait-il acceptable de renoncer à mener des recherches dans des pays dépourvus de moyens identiques à ceux en vigueur dans les centres hospitalo-universitaires les plus renommés?

Des enjeux spécifiques s'imposent dans le champ de la recherche vaccinale. Ils sont parfaitement caractérisés dans un document réalisé par l'ONUSIDA en 2001 [27] et nous confrontent nécessairement aux dilemmes éthiques que Daniel Hoth exposait dès 1992 [28]: «Il ne faut pas attendre un vaccin idéal. [...] Nous devons nous souvenir constamment que la recherche ne s'effectue pas dans le vide, et que l'épidémie fait rage. Nous devons orienter nos recherches en considérant non seulement les meilleurs principes scientifiques mais notre attention aiguë à l'urgence pratique liée au problème ».

C'est sur le concept d' « urgence pratique » que l'on peut conclure cette approche. Le VIH-sida doit se comprendre comme un défi dont on ne peut atténuer ni la violence, ni la gravité. II nous situe, bien souvent malgré nous, là où nous ne pouvions penser que devaient s'exercer nos devoirs d'humanité. On peut toutefois constater que cette pandémie a suscité et édifié de nouvelles formes de solidarités qui ont bouleversé nos repères en termes de soins et de santé publique. Ces quelques acquis des années sida éclairent autrement la figure de la personne malade et nous permettent de comprendre avec d'autres références la dimension politique de la santé publique. De telle sorte qu'il semble plus que jamais indispensable de témoigner davantage que de l'attention aux personnes qui revendiquent le droit de vivre au-delà du sida.

«Des personnes ont refusé d'accepter l'inacceptable, alors qu'elles savaient dans quelle logique de mort pouvait s'intégrer leur dessein d'action. [...] D'autres besoins humains, d'autres espoirs ont été reconnus à travers la vie ». Jonathan M. Mann, 1998 [29]. $\diamond$

\section{SUMMARY}

HIV-AIDS: ethics in healthcare, research, and access to treatments

Violence and gravity cannot be ignored when considering HIV and AIDS challenges. However, coping with this pandemic disease caused us to build up and experience new ways of solidarity that have transformed our views of medical care and public health. The few achievements of these years throw a new light on the figure of the ill person. They brought new references allowing a different understanding of the political stakes of public health. Partnership or even therapeutic alliance are now a framework to the understanding of respect, solidarity and equity. New viewpoints exist on the health care relationship. Our achievements show at first injustice in the access of treatments at the international level. They make clear our moral responsibilities toward every person that require a treatment. This idea should be emphasized when applied on the most vulnerable communities. The most urgent challenges that remain today are extreme precariousness situations, treatment access conditions, information, and quality of life. $\diamond$ 


\section{RÉFÉRENCES}

1. DGS. Circulaire DGS/PGE/lC, $n^{\circ} 85$, du 20 janvier 1988, relative à la mise en place d'un dispositif de dépistage anonyme et gratuit du virus de l'immunodéficience humaine (complétée par la Circulaire DGS/PGE/IC, $n^{\circ} 311$, du 30 mars 1988).

2. OMS. Droits de l'homme et sida: la discrimination entrave la lutte contre le sida. Communiqué $0 M S / 48$, 22 juin 1993. Se référer à Le $\mathrm{VIH}$ et les droits de l'homme. Directives internationales, HCDH, ONUSIDA, 1998.

3. Borillo D. Le sida contre les droits de l'homme? Réflexion juridique sur I'infection par le VIH. Agora 1991; 18-19: 27.

4. Benevise N. Journal d'une infirmière. Paris: Plon, $1993: 140$.

5. Evin C. Présentation du plan de 2 ans de l'Agence française de lutte contre le sida, 29 janvier 1990.

6. Got C. Rapport sur le sida. Paris: Flammarion, 1989 : 132.

7. Vivre le sida. Le livre blanc des États Généraux des 17 et 18 mars 1990 à Paris. Paris: Cerf, 1992.

8. 1001 façons de vivre avec son traitement. Vivre avec le sida. Paris: Ramsay, 1999.

9. Les malades prennent la parole. Paris: Ramsay, 1999.

10. Augé M. La société, le sida et le diable. Entretien avec Guitta Pessis-Pasternak, Le Monde, 20 décembre 1988.

11. Defert D. Au front. Le Monde, $1^{\text {er }}$ décembre 1988.

12. Kazatchkine M. La consultation du soir. Un témoignage engagé sur cette épidémie qui bouleverse le monde. Paris: Presses de la Renaissance, 2003.

13. Barzach M. Le paravent des égoïsmes. Paris: Odile Jacob, 1989 : 106.

14. René L. Dans le respect de la personne humaine. Bulletin de l'Ordre des Médecins $1991 ; n^{\circ} 9$.

15. Mann JM. OMS. Séance inaugurale de de la Ve
Conférence internationale sur le sida, Montréal, 4 juin 1989.

16. Children on the Brink 2002. A joint report on orphan estimates and program strategies. UNAIDS, UNICEF, USAID, juillet 2002.

17. Report on the global HIV/AIDS epidemic. UNAIDS, 2002.

18. Assemblée générale des Nations-Unies, Session extraordinaire sur le VIHsida, juin 2001, résolution paragraphe 55.

19. Curran JW. Senior Lecture, XIV Conférence internationale sur le sida. Barcelone, 11 juillet 2002.

20. Association médicale mondiale (AMM). Déclaration d'Helsinki, 1964-2000, article 8.

21. Beyrer C, Kass NE. Human rights, politics, and reviews of research ethics. Lancet 2002; 360: 246-51.

22. Rapport Belmont. Commission nationale pour la protection des sujets humains de la recherche biomédicale et comportementale, ÉtatsUnis, 1978.

23. Brody $B A$. Ethical issues in clinical trials in developing countries. Stat Med 2002; 21: 2853-8.

24. OMS. Lignes directrices opérationnelles pour les Comités d'éthique chargés de l'évaluation de la recherche biomédicale. 2000.

25. ANRS. Charte d'éthique de la recherche dans les pays en développement. Mai 2002, articles 1.3, 1.4 et 1.5 .

26. Avis sur les problèmes éthiques posés par la recherche clinique dans les pays en développement. Conseil national du sida, 11 mars 2003.

27. ONUSIDA. Considérations éthiques dans la recherche de vaccins préventifs contre le VIH. Septembre 2001.

28. Hoth D. National institute of allergy and infectious diseases, NIH, États-Unis, VIII ${ }^{e}$ Conférence

\section{DÉCLARATION UNIVERSELLE DES DROITS DES MALADES DU SIDA ET DES SÉROPOSITIFS}

Remise à Michèle Barzach (ministre de la Santé) par le Pr Alain Deloche, président de l'association Médecins du Monde, et par Daniel Defert, président de l'association AIDES, le 16 octobre 1987.

Le sida est maladie et symptôme de notre époque. En dépit de son actuelle gravité, en dépit de l'absence momentanée de tout traitement efficace, et au-delà des réactions médiatiques, politiques ou simplement humaines que la peur suscite, le sida reste une maladie semblable aux autres que la médecine a déjà maîtrisées et qu'elle maîtrisera.

En dehors des rapports sexuels et de la transmission par voie sanguine, les malades ne représentent aucun risque de contagion.

L'information et l'éducation du public sont actuellement les conditions nécessaires à l'acquisition par chacun des gestes de prévention seuls susceptibles d'enrayer l'épidémie. Elles sont donc garantes de la santé et de la liberté des populations.

Cette situation impose sagesse, exige le respect scrupuleux d'une éthique, dicte des impératifs (il en existe 10):

1. Au regard de la loi et de la médecine, le sida est une affection comme les autres.

2. Les personnes atteintes par le virus sont protégées par la loi commune. Aucune loi d'exception ne pourra leur être appliquée.

3. Les soins aux personnes atteintes par le virus doivent être consentis sans aucune restriction.

4. Les transfusions sanguines et l'utilisation du matériel de prise de sang et d'injection devront offrir toutes les garanties possibles d'innocuité. Les responsables de chaque pays ont droit pour cela à la coopération internationale.

5. Nul n'a le droit de restreindre la liberté ou les droits des personnes au seul motif qu'elles sont atteintes par le virus, quels que soient leur race, leur nationalité, leur religion, leur sexe ou leur orientation sexuelle.

6. Toute référence à la maladie présente ou future faite sans le consentement de la personne atteinte par le virus doit être considérée comme préjudiciable et sanctionnée dans le cadre du droit commun.

7. Toute action tendant à refuser aux personnes porteuses de virus un emploi, un logement, une assurance, ou à les en priver, à restreindre leur participation aux activités collectives, scolaires et militaires doit être considérée comme discriminatoire et sanctionnée.

8. En aucun cas des examens de dépistage du virus doivent être pratiqués à l'insu des personnes.

9. Tous les examens de contrôle ou les dépistages qui s'avèrent nécessaires doivent être accomplis dans le respect de l'anonymat et couverts par le secret médical. Aucun dépistage ne peut être proposé qui ne soit accompagné de la garantie d'un soutien psychologique, médical et social.

10. Le secret médical qui lie les médecins entre eux et chaque médecin à son patient doit être absolu, notamment à l'égard des employeurs et des services publics. II ne doit connaître aucune exception, quelles que soient les exigences de la technologie médicale moderne. Les données recueillies par le médecin ne doivent servir qu'à des fins médicales. Tout manquement à cette déontologie devra être poursuivi et donner lieu à réparation.

internationale sur le sida,

Amsterdam, juillet 1992.

29. Mann JM. Ne pas oublier ce qui nous unit. Le Journal du sida 1998; 107.

\section{TIRÉS À PART}

દ. Hirsch 